Article

\title{
Design of Concentric Ring Antenna Arrays Based on Subarrays to Simplify the Feeding System
}

\author{
Elizvan Juárez ${ }^{1} \oplus$, Marco A. Panduro ${ }^{1, *} \mathbb{C}$, Alberto Reyna ${ }^{2} @$, David H. Covarrubias ${ }^{1}$, \\ Aldo Mendez ${ }^{2}$ and Eduardo Murillo ${ }^{3}$ \\ 1 Electronics and Telecommunications Department, CICESE Research Center, Carretera Ensenada-Tijuana \\ No. 3918, Zona Playitas, Baja California, Ensenada 22860, Mexico; elizvan@cicese.edu.mx (E.J.); \\ dacoro@cicese.mx (D.H.C.) \\ 2 Electronics Department, Universidad Autónoma de Tamaulipas, UAMRR-R, Carretera Reynosa-San \\ Fernando, Tamaulipas, Reynosa 88779, Mexico; alberto.reyna@docentes.uat.edu.mx (A.R.); \\ almendez@uat.edu.mx (A.M.) \\ 3 Centro de Nanociencias y Nanotecnología, UNAM, Baja California, Ensenada 22860, Mexico; \\ emurillo@cnyn.unam.mx \\ * Correspondence: mpanduro@cicese.mx
}

Received: 24 April 2020; Accepted: 4 June 2020; Published: 8 June 2020

\begin{abstract}
The global synthesis for antenna arrays that produce a desired radiation pattern is a scientific symmetry problem. This paper deals with the design of concentric ring antenna arrays to simplify the feeding system using a cophasal subarray configuration. This simplification in the feeding network is achieved by the reduction of phase shifters via a proper clustering of radiators applying one amplitude and one phase excitation by subarray in the array system. The array design for this geometry considers an optimization process based in differential evolution to reduce the side-lobe level, resulting in simplification of the feeding network. Simulation results based on CST Microwave Studio and HFSS are provided to verify the accuracy of the array model and to take mutual coupling into account. These simulations are provided using a circular patch as antenna element to generate a scannable array pattern over the elevation plane. Furthermore, an analysis of the manufacturing tolerances was made to verify the reliability of our design.
\end{abstract}

Keywords: antenna array; concentric rings geometry; subarrays; beam steering; phase shifter

\section{Introduction}

The number of devices connected to mobile networks is continually increasing with the use of wireless sensors. One promising solution for overcoming the associated issues is the use of phased antenna arrays at the hardware level of the sensor nodes. The applications of phased array systems are well known and widespread in numerous telecommunications fields. Phase shifters are essential components and are extensively used in modern phased-array antennas [1]. However, with the complexity in the feeding network and its interaction with the radiating elements, the design of phased arrays can be a challenging problem [2,3]. Design cases using a large number of phase shifters can be particularly complicated. In conventional antenna arrays, the beamforming network (with phase and amplitude coefficients) uses one phase shifter (and, often, one phase shifter and one attenuator) per antenna element. In these cases, the beamforming network can be highly complex and limited for applications with a large number of antenna elements.

It is desirable in all applications to ensure low complexity and low cost by using a small number of phase shifters to accomplish the requirements of scanning and side-lobe level. A significant amount of previous literature relates to the reduction of the number of phase shifters. Notably, simplification of 
the complexity of the feeding system has been undertaken for a large number of applications: mobile earth stations [4], radar systems [5-7], vehicular uses [8] and autonomous systems [9].

The conventional method of reducing the number of phase shifters is to divide the array into uniform subarrays (or groups) and feed each group with a single-phase shifter [10-12]. However, grating lobes appear in the visible space and cause the side-lobe level to increase abruptly $[13,14]$. This problem has been dealt in previous work by using overlapping subarrays [15-20], aperiodic subarrays [4,21], random feeding networks [2] and interleaved subarrays [3]. The technologies of overlapping subarrays and random feeding networks have been applied recently [2,20].

However, the majority of applications based on overlapping subarrays and random feeding networks consider limited-scan arrays for conventional antenna array structures, such as linear and planar. The application of subarrays to reduce the number of phase shifters in array structures of wide-angle scanning is scarce.

The current paper introduces the design of concentric ring antenna arrays based on subarrays to simplify the feeding system. The choice of concentric ring geometry is not arbitrary. This array structure was chosen considering its applications in technology, including mobile communication, radar system, satellites and medical treatment. In addition, the behavior of the cophasal excitation of this structure facilitates the generation of subarrays.

This paper proposes the design of concentric ring antenna arrays using a cophasal subarray configuration. This array design configuration using a structure of concentric rings can provide desired radiation characteristics with angular scanning and reduces the number of phase shifters employed in the system.

Because the geometry influences the results, the design methodology for this array geometry considers an optimization process based on differential evolution [22] to reduce the side-lobe level, resulting in the simplification of the feeding network. The reduction of phase shifters is achieved via a proper clustering of radiators applying one amplitude and one phase excitation by subarray in the array system.

This paper illustrates a highly nonlinear symmetry problem related to the design of antenna arrays using concentric rings geometry for reducing the number of phase shifters and achieving maximum performance. The novelty of the paper concerns the proposed subarraying technique to reduce phase shifters using the specified array geometry. Amongst the current state-of-the-art approaches, there appears to be no trace of works dealing with the design of antenna arrays to reduce the number of phase shifters using the proposed technique and concentric rings geometry.

Furthermore, simulation results based in CST Microwave Studio and HFSS are provided to verify the accuracy of the array model and to take mutual coupling into account. The CST Microwave Studio simulations consider a circular patch as antenna element to generate a scannable array pattern over the elevation plane.

\section{Array Pattern with Cophasal Subarrays}

The approach proposed in this paper uses a concentric ring antenna array to generate a radiation pattern over the elevation plane, and to reduce the number of phase shifters employed by applying a cophasal subarray configuration, i.e., for grouping the elements using one amplitude and one phase excitation by each cophasal subarray in the array system.

The array factor of the concentric ring antenna array (see Figure 1) can be expressed as [23,24]:

$$
A F=\sum_{n=1}^{N_{r}} \sum_{m=1}^{N_{n}} I_{n m} \exp \left[j\left(k r_{n} \sin \theta \cos \left(\varphi-\varphi_{n m}\right)+\alpha_{n m}\right)\right]
$$

where $N_{r}$ is the number of circular rings in the array, $N_{n}$ is the number of antenna elements in the $n$th circular ring, $I_{n m}$ and $\alpha_{n m}$ are the amplitude and phase excitations of the $m$ th antenna element for the $n$th circular ring, respectively, $r_{n}$ is the radius of $n$th circular ring, $k$ is the propagation number, $\theta$ is 
the elevation angle and $\varphi$ is the azimuth angle. The main beam is scanned to $\left(\theta_{0}, \varphi_{0}\right)$ by applying a cophasal excitation, therefore, $\alpha_{n m}$ is given by:

$$
\alpha_{n m}=-k r_{n} \sin \theta_{0} \cos \left(\varphi_{0}-\varphi_{n m}\right)
$$

The total radiation pattern of the complete system, $P(\theta, \varphi)$, is determined as:

$$
P(\theta, \varphi)=A F(\theta, \varphi) \times E P(\theta, \varphi)
$$

where $E P(\theta, \varphi)$ is the pattern of the antenna element.

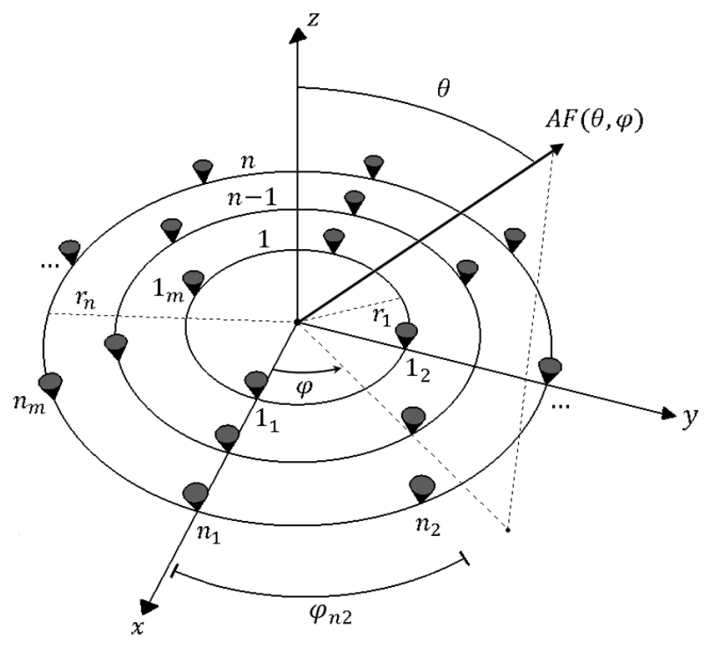

Figure 1. Concentric ring antenna array formed by $N_{r}$ circular rings.

In order to explain the proper clustering of radiators, Figures 2 and 3 show a concentric ring antenna array of three rings with $N_{1}=4, N_{2}=6$ and $N_{3}=8$. This configuration uses seven subarrays created strategically considering cophasal excitation. The behavior of the cophasal excitation values in the elevation plane based on Equation (2) is illustrated in Figure 4. This figure illustrates the phase value required by each antenna element to scan the main beam in $\theta_{0}=\left[-40^{\circ}, 0^{\circ}, 20^{\circ}, 40^{\circ}, 60^{\circ}\right]$ in the elevation plane in the cut of $\varphi=\pi$. This behavior for this array geometry illustrates that one same phase value (elements with similar phase values) can be assigned to several antenna elements, as shown in Figure $4 \mathrm{~b}$. This can be taken as an advantage for grouping the antenna elements in subarrays and reducing the number of phase shifters employed in the antenna system. Therefore, the groups or subarrays are set considering the cophasal excitation required for scanning the main beam; then, this subarray configuration can be named cophasal subarrays configuration. In the case of Figure 2, the amplifiers can be considered variable or fixed during beam scanning. Figure 3 illustrates the subarray configuration proposed for the case of using a distribution of raised cosine amplitude excitation [2].

This amplitude distribution has been shown to be efficient in antenna arrays for the amplitude excitation function [2]. This design case can be useful to evaluate the performance of the optimized case. The case of raised cosine distribution needs 12 (fixed) amplifiers to generate the amplitude excitations across the antenna array [2] for that design configuration, as shown in Figure 3. As shown in the phase values of Figure $4 \mathrm{a}$, four antenna elements $\left\{n_{2}, n_{4}, n_{13}, n_{17}\right\}$ represent zero phase values for all scanning directions. In this case, these antenna elements do not require a phase shifter (or these antenna elements could be set in a subarray fed by one phase shifter). The next subarrays that present the same phase value (or similar values) are groups of three elements, the cases of $\left\{n_{1}, n_{6}, n_{10}\right\},\left\{n_{3}\right.$, $\left.n_{7}, n_{9}\right\},\left\{n_{5}, n_{12}, n_{18}\right\}$ and $\left\{n_{8}, n_{14}, n_{16}\right\}$, and finally two groups of one antenna element: $\left\{n_{11}\right\}$ and $\left\{n_{15}\right\}$. The power delivery network can be considered by using a cascaded unequal dividers network [2] 
(usually with Wilkinson divisors). The power and phase in each element of a same subarray (in our configuration of cophasal subarrays) must be equal. This is achieved by using 1-to-3 and 1-to-4 equal power dividers.

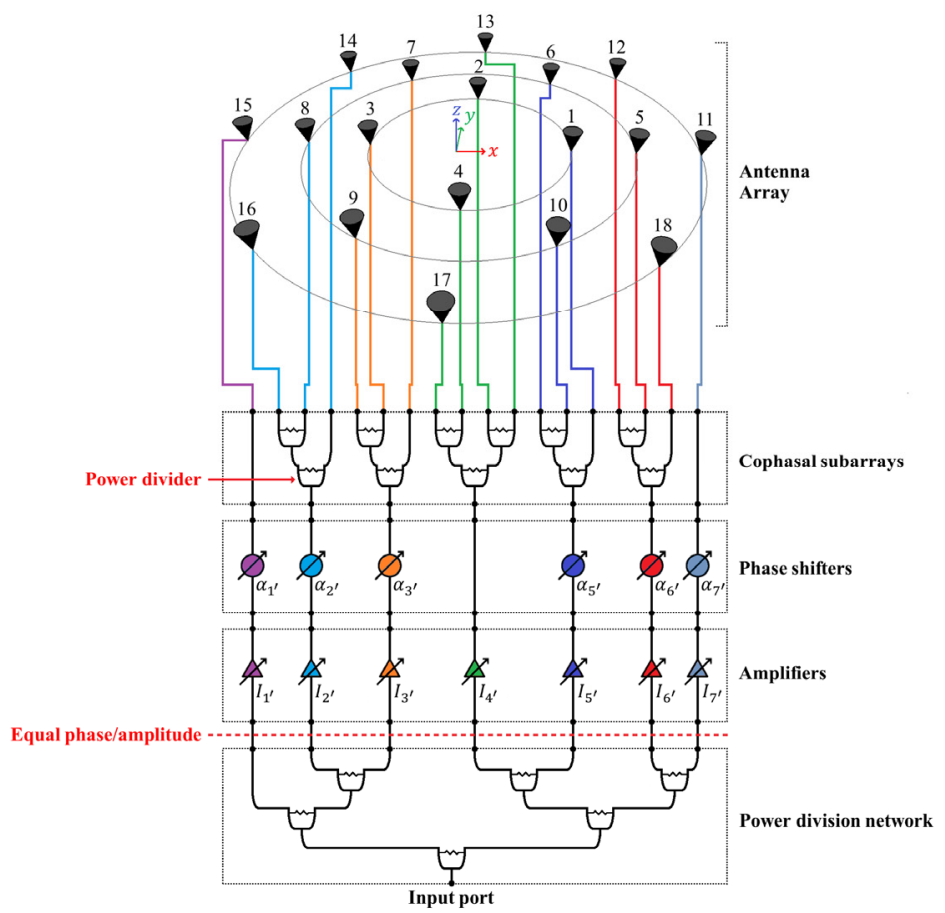

Figure 2. Subarrays configuration in a concentric ring antenna array ( 3 rings configuration with $r_{1}=0.5 \lambda, r_{2}=1.00 \lambda$ and $\left.r_{3}=1.52 \lambda\right)$ to scan the main beam in the elevation plane.

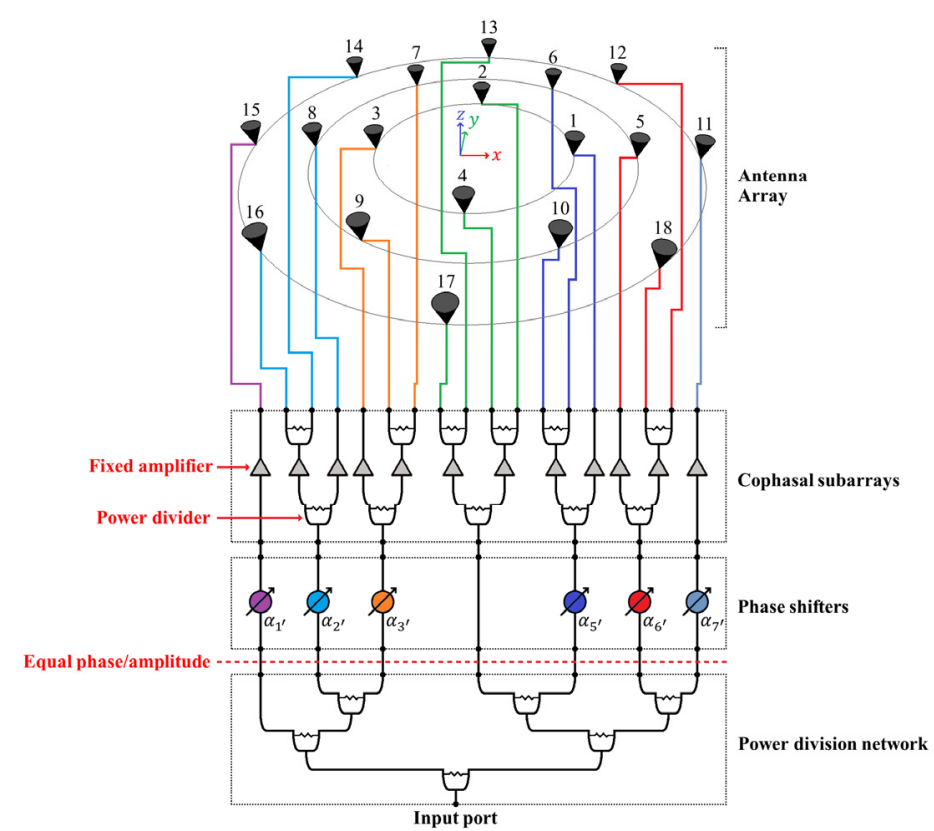

Figure 3. Previous subarrays configuration for the case of using a distribution of raised cosine amplitude excitation with fixed amplifiers. 


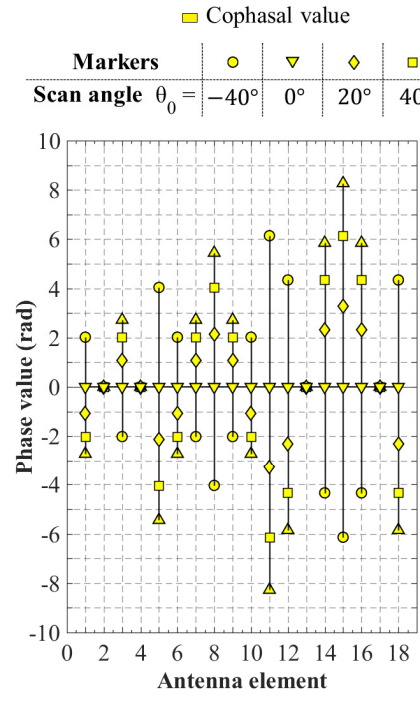

(a)

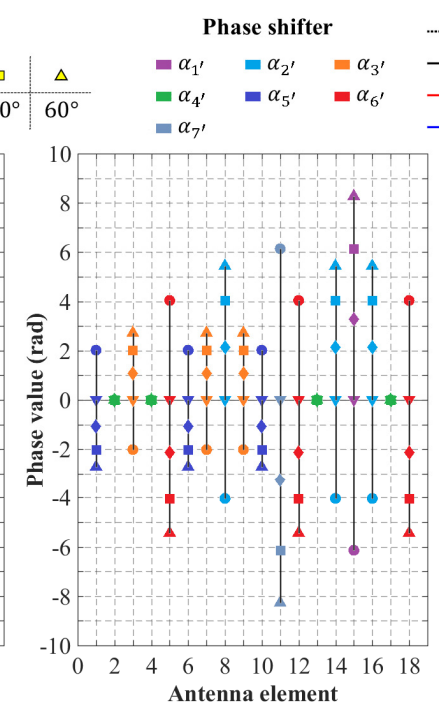

(b)
-... $\theta_{0}=-40^{\circ}$ without cophasal subarrays (18 PSs)
$-\theta_{0}=40^{\circ}$ without cophasal subarrays (18 PSs)
$-\theta_{0}=-40^{\circ}$ using cophasal subarrays (6 PSs)
$-\theta_{0}=40^{\circ}$ using cophasal subarrays (6 PSs)

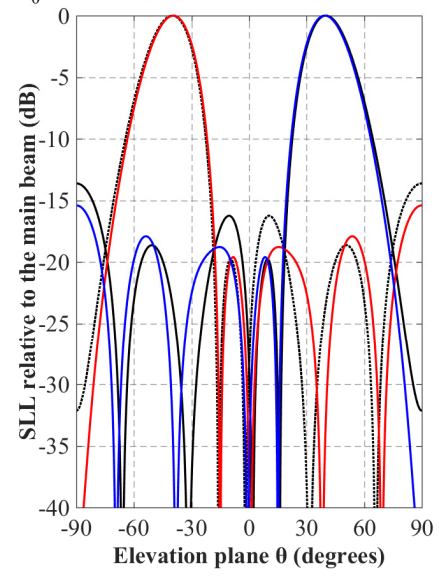

(c)

Figure 4. (a) Phase values calculated using Equation (2), (b) phase values obtained using cophasal subarrays configuration in $\theta_{0}=\left[-40^{\circ}, 0^{\circ}, 20^{\circ}, 40^{\circ}, 60^{\circ}\right]$; (c) Array factor comparison using cophasal subarrays.

Note that a scannable pattern (see Figure 4 ) in the range of $-40^{\circ} \leq \theta_{0} \leq 40^{\circ}$ (with a peak side-lobe level of $-15 \mathrm{~dB}$ ) could be generated maintaining the same subarray configuration, i.e., by just varying the value of the phase shifter for each subarray: $\alpha_{1^{\prime}}, \alpha_{2^{\prime}}, \alpha_{3^{\prime}}, \alpha_{5^{\prime}}, \alpha_{6^{\prime}}, \alpha_{7^{\prime}}$. This configuration represents the case of using the radii values of $r_{1}=0.5 \lambda, r_{2}=1.00 \lambda$ and $r_{3}=1.52 \lambda$. These radii values were obtained using our previous experience in antenna arrays optimization [25-30]. Although it is obvious that different array configurations and scanning angle (such as different radii values) result in different element phase distribution, the same subarray configuration will be retained for a specific array configuration and scanning angle range. This condition is retained because the phase values for each subarray are assigned in a perpendicular way to the scanning elevation plane ( $x$-z plane), i.e., although the phase value changes for each scanning direction the same groups or subarrays are obtained. As commented previously, this is the reason why we chose concentric rings as the array geometry. The behavior of the cophasal excitation of this structure facilitates the generation of subarrays.

The decision variables for this design case are the values of the amplitude and phase excitations feeding each subarray. In our optimization procedure, the optimization goal is to minimize the side-lobe level. The technique of differential evolution was considered based on its versatility for designing antenna arrays and our previous experience. Differential evolution is a well-known state-of-the-art technique; details and implementation procedures can be found in $[22,26]$.

\section{Results and Discussion}

The method of differential evolution was implemented in MATLAB for optimizing the values of the amplitude and phase excitations using the cophasal subarray configuration for grouping the elements illustrated in Figure 2 (considering $N_{1}=4, N_{2}=6$ and $N_{3}=8$ ). Differential evolution was applied to find the values of the amplitude and phase excitations to generate a scannable array pattern over the elevation plane. We followed the literature and our previous results to set the parameters for the optimization algorithm. We considered the design cases of variable and fixed amplifiers. In addition to these design cases, we make a comparison to the case in which a raised cosine distribution (Figure 3) across the antenna array is used. 


\subsection{Array Factor Results}

The first case studied using cophasal subarrays was that considering the response of the array factor. In this case, Figure 5 illustrates the behavior of the side-lobe level obtained (for the array factor optimized by differential evolution) versus scanning direction for the concentric ring antenna array using cophasal subarrays with optimization using variable and fixed amplifiers, and its comparison with respect to the design cases using a raised cosine distribution and uniform amplitude excitation. The behavior of the side lobe level as a function of scanning direction in steps of $5^{\circ}$ is illustrated. As shown in Figure 5, the optimized case using differential evolution and cophasal subarrays with variable amplifiers provides better side-lobe level values for all of the scanning range. The important aspect is that this side lobe-level behavior in the scanning range is achieved by using only six phase shifters (and seven variable amplifiers), reducing the number of phase shifters from 18 for a conventional array (which uses one phase shifter per antenna element without optimization) to six. This represents a $66.6 \%$ reduction in the number of phase shifters used in the array system at the expense of using seven variable amplifiers. The highest or maximum value of the side-lobe level was $-26.87 \mathrm{~dB}$ found for the array factor in the case of $\theta_{0}=30^{\circ}$. The minimum value of side-lobe level (in the scanning range) was $-20.29 \mathrm{~dB}$ found in $\theta_{0}=40^{\circ}$. There was a reduction in the side-lobe level of almost $10 \mathrm{~dB}$ with respect to the conventional array of cophasal excitation, and almost $8 \mathrm{~dB}$ with respect to the case using cophasal subarrays without optimization. The optimized case of cophasal subarrays provides a scanning range over the elevation plane of $\left[-45^{\circ}, 45^{\circ}\right]$ for a peak side-lobe level below $-15 \mathrm{~dB}$, and a range of $\left[-50^{\circ}, 50^{\circ}\right]$ for a peak side lobe level of $-10 \mathrm{~dB}$.

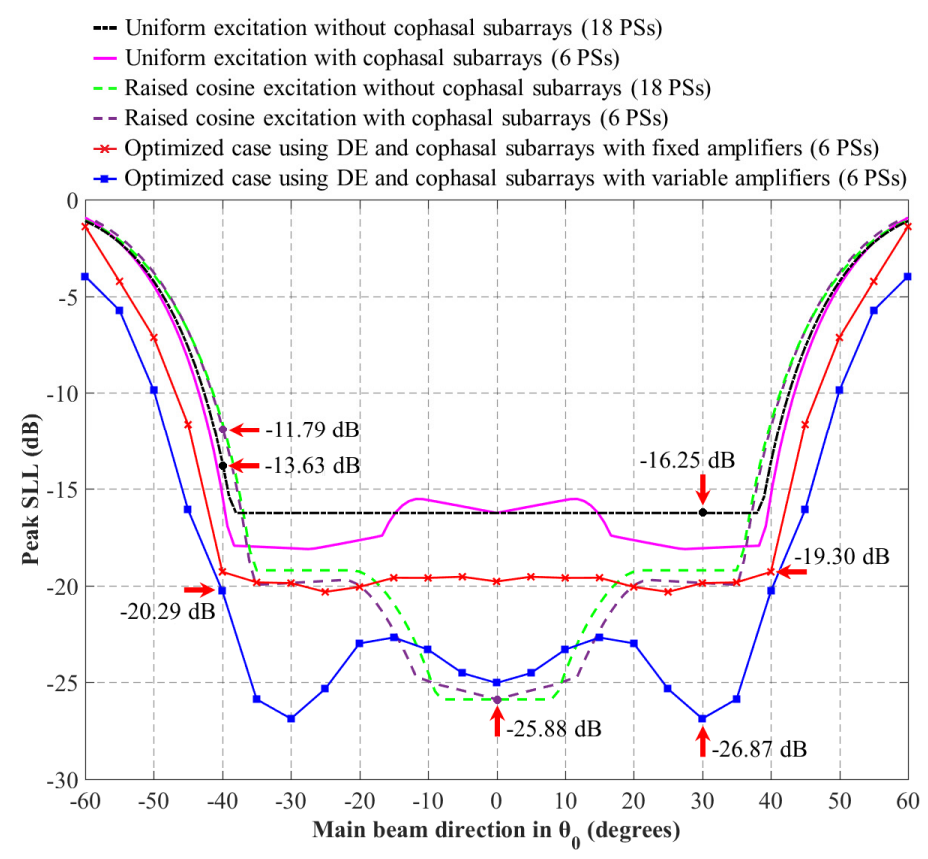

Figure 5. Behavior of the side lobe level versus scanning direction for the concentric ring antenna array using cophasal subarrays with optimization (variable and fixed amplifiers) and its comparison with respect to the other design cases.

The optimized case using fixed amplifiers provides a good design compromise. This design case provides a range of scanning of $\left[-40^{\circ}, 40^{\circ}\right]$ for a peak side-lobe level of $\cong-20 \mathrm{~dB}$, and a range of $\left[-45^{\circ}\right.$, $45^{\circ}$ ] for a peak side lobe level of $\cong-12 \mathrm{~dB}$. It is important to note that this performance is obtained using six phase shifters and seven fixed amplifiers, holding them constant during the beam scanning, and avoiding the use of variable amplifiers. The side-lobe level response is much flatter than the variable amplifier case and the other cases. 
Furthermore, it is interesting to note that the case of raised cosine distribution presents similar values of side-lobe level using six or 18 phase shifters. This design case provides a range of scanning of $\left[-10^{\circ}, 10^{\circ}\right]$ for a peak side-lobe level of $\cong-25 \mathrm{~dB}$, a range of $\left[-35^{\circ}, 35^{\circ}\right]$ for a peak side-lobe level of $\cong-20 \mathrm{~dB}$ and a range of $\left[-40^{\circ}, 40^{\circ}\right]$ for a peak side-lobe level of $\cong-11 \mathrm{~dB}$. This design case requires 12 fixed amplifiers to adequately generate the amplitude distribution. Depending on the designer criteria this design case could be a good option for cases of limited scanning range.

Figure 6 shows some example cases of an array factor optimized with differential evolution and cophasal subarrays using variable and fixed amplifiers, and its comparison with respect to the other design cases, for scanning directions of (a) $\theta_{0}=0^{\circ}$, (b) $\theta_{0}=30^{\circ}$ and (c) $\theta_{0}=40^{\circ}$. The method of differential evolution efficiently computes the values of the design variables to generate an array factor with characteristics of low side-lobe level without pattern distortion during beam steering.

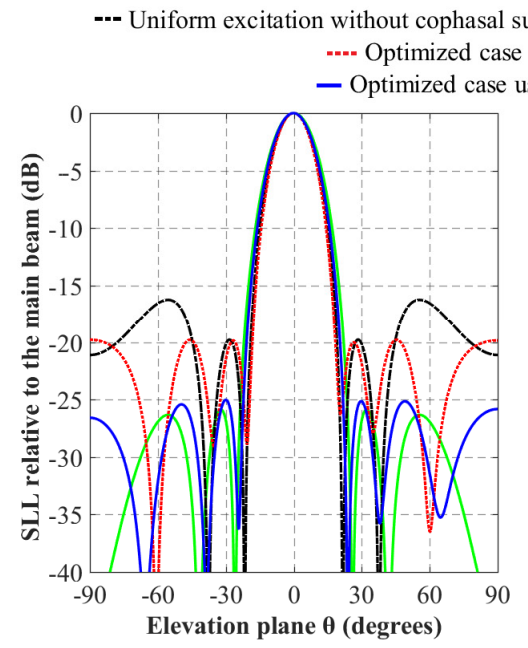

(a)

- Raised cosine excitation with cophasal subarrays (6 PSs) subarrays with fixed amplifiers (6 PSs)

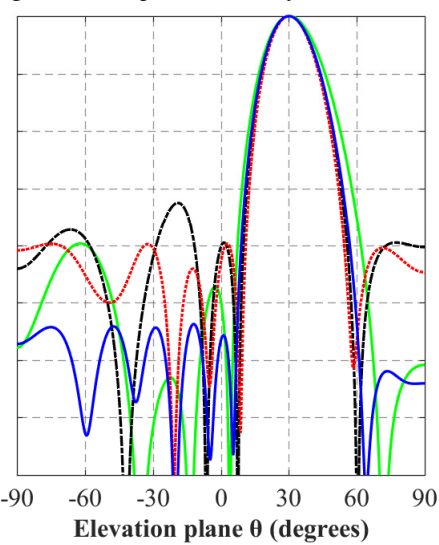

(b)

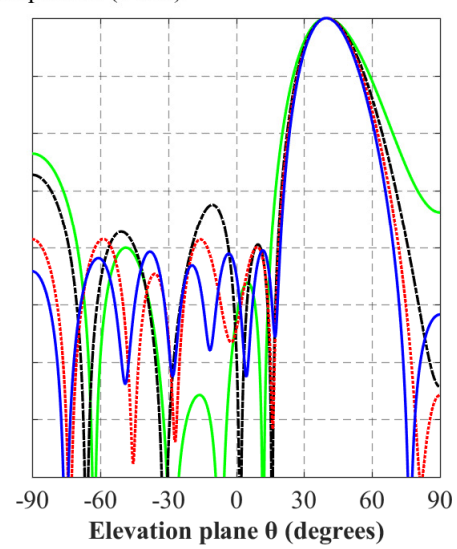

(c)

Figure 6. Array factor optimized with differential evolution and cophasal subarrays using variable and fixed amplifiers and its comparison with respect to the other design cases for scanning directions of (a) $\theta_{0}=0^{\circ}$, (b) $\theta_{0}=30^{\circ}$ and (c) $\theta_{0}=40^{\circ}$.

The values of the amplitude and phase excitations for each cophasal subarray were determined by differential evolution for each scanning direction in steps of $5^{\circ}$ for the optimized case using variable amplifiers. In the case of fixed amplifiers, the same set of amplitude excitations was held constant during beam scanning. Figure 7 shows the values of the amplitude and phase excitations for the optimized cases using variable and fixed amplifiers with scanning directions of $\theta_{0}=30^{\circ}$ and $\theta_{0}=40^{\circ}$. The minimum value of the amplitude excitation was 4.46 and the maximum value was 10.98 for the case of variable amplifiers, and 7.18 and 13.66, respectively, using fixed amplifiers. In this case, the amplitude weights could be realized in a real hardware setup rescaling all the complex coefficients before applying them to a real system. In a real system, the amplitudes will be adjusted at every layer in such a way that the conservation law of the energy will be preserved. Furthermore, it is interesting to note that the values of phase excitations obtained follow the behavior of a phase slope, i.e., a progressive phase excitation is obtained similar to phase excitation for linear arrays. This is because the phase values for each subarray are assigned in a perpendicular way to the scanning elevation plane $(x-z$ plane). 

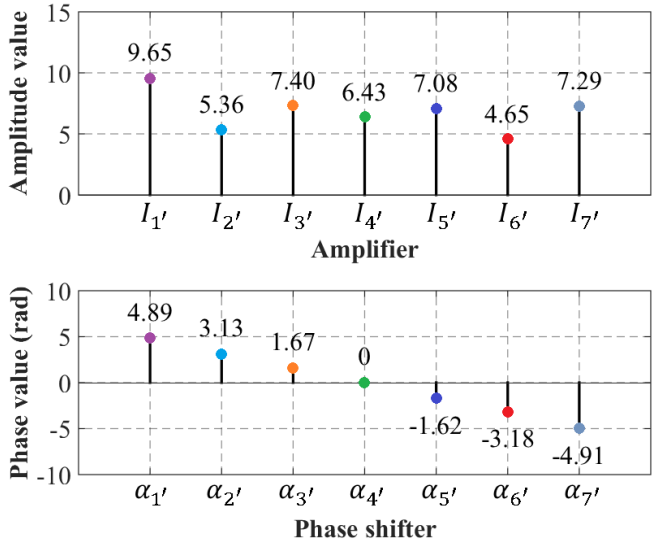

(a)
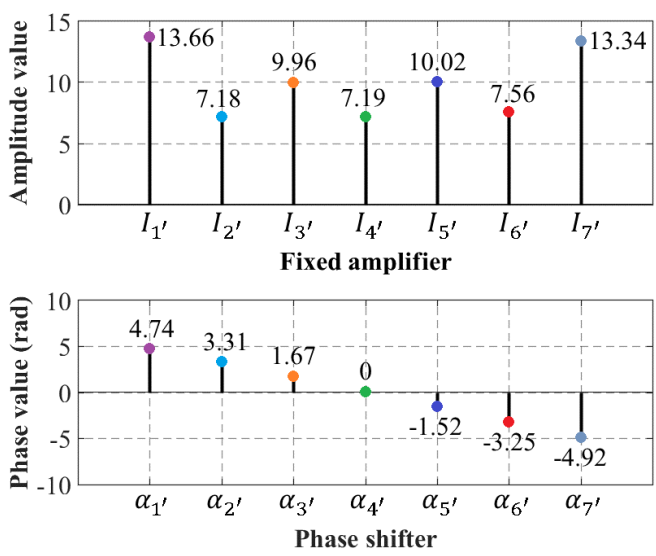

(c)
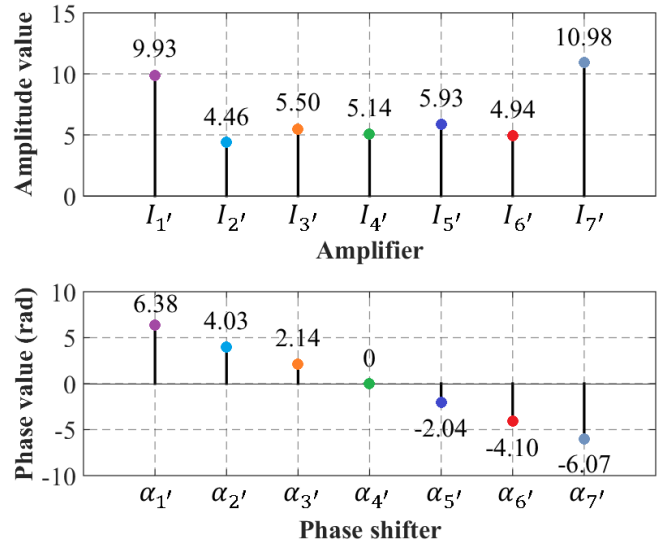

(b)
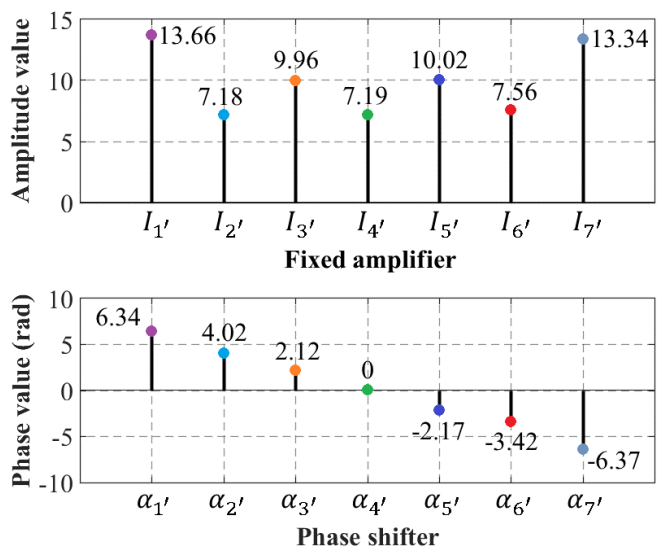

(d)

Figure 7. Values of the amplitude and phase excitations for the optimized cases using variable $\left((\mathbf{a}) \theta_{0}=\right.$ $30^{\circ}$ and (b) $\left.\theta_{0}=40^{\circ}\right)$ and fixed $\left((\mathbf{c}) \theta_{0}=30^{\circ}\right.$ and (d) $\left.\theta_{0}=40^{\circ}\right)$ amplifiers.

\subsection{CST Microwave Studio Simulation}

In order to verify the accuracy of the array model and to take mutual coupling into account, the optimized design case was simulated in full-wave using CST Microwave Studio. The CST Microwave Studio simulations were run in a high performance server consisting of several GPU cards to accelerate the process. The optimized design case using variable amplifiers was modelled and implemented in CST Microwave Studio using a circular patch as antenna element for an operation frequency of 2.4 GHz with the dimensions: $a=17.27 \mathrm{~mm}, h=1.6 \mathrm{~mm}$ and $p^{\prime}=6.37 \mathrm{~mm}$ [31]. The substrate of the circular patch presents characteristics of relative permittivity $\varepsilon_{\mathrm{r}}=4.2, \mu=1$ and 0.025 of tangential losses, corresponding to FR4 values. We considered full-wave analysis of the feeding network for the phase and amplitude values found in the optimized design (without post-processing the results of the full-wave analysis of the antenna array).

First, we examined the active impedance or active $S$ parameters of the array design. The behavior of the reflection coefficient changes during beam scanning, so we considered a scanning direction far from the natural radiation response of the array (i.e., in $\theta_{0}=-40^{\circ}$ ). The active reflection coefficient (or scan reflection coefficient) is the reflection coefficient for a single antenna element in the array antenna, in the presence of mutual coupling. The active reflection coefficient is a function of frequency, scanning direction and the excitation of the neighboring elements [32]. Every scanning direction was examined (of the scanning range) far from the natural radiation response of the array. This was challenging, especially for the farthest directions (from the natural radiation response of the array), 
but the active reflection coefficient of 18 antenna elements remained below $-10 \mathrm{~dB}$ for all scanning directions $\left[-40^{\circ}, 40^{\circ}\right]$ at $2.4 \mathrm{GHz}$.

Figure 8 shows the active reflection coefficient of 18 antenna elements at $2.4 \mathrm{GHz}$ corresponding to the scanning direction of the main beam in $\theta_{0}=-40^{\circ}$. As was expected, the elements show a good matching performance. Furthermore, the reflection coefficient of elements is lower than $-10 \mathrm{~dB}$ in the frequency of interest, which is acceptable for many applications. This behavior remained around the central frequency of the array from 2.37 to $2.41 \mathrm{GHz}$ with $40 \mathrm{MHz}$ of bandwidth.

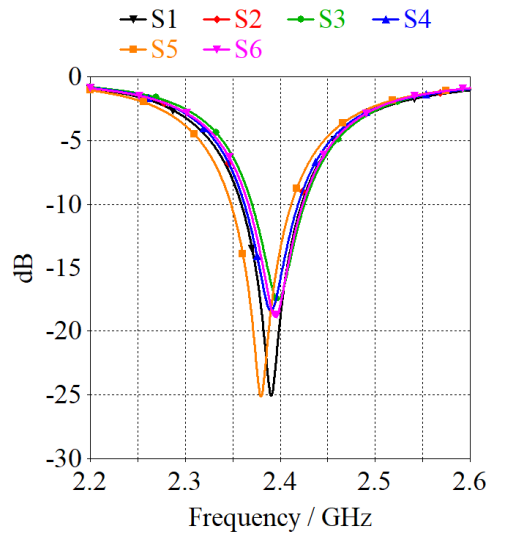

(a)

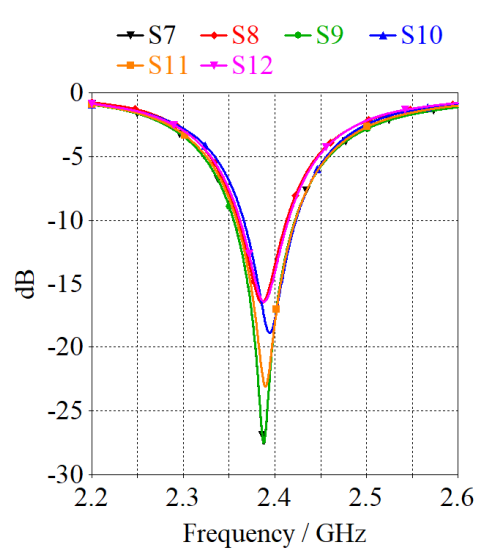

(b)

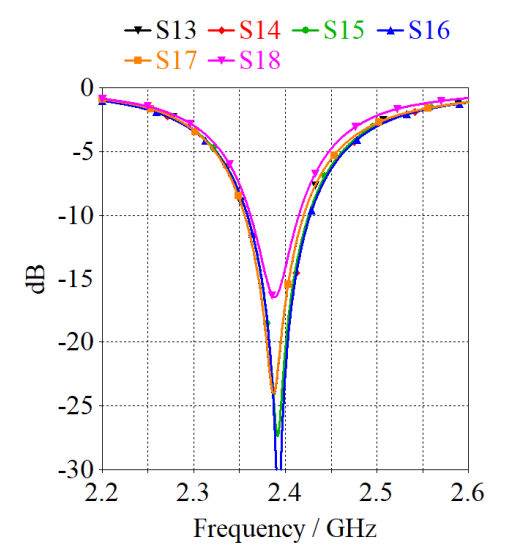

(c)

Figure 8. Active reflection coefficient of all elements in the array with scanning direction in $\theta_{0}=-40^{\circ}$ : (a) $[\mathrm{S} 1, \mathrm{~S} 2, \ldots, \mathrm{S} 6]$, (b) $[\mathrm{S} 7, \mathrm{~S} 8, \ldots, \mathrm{S} 12]$ and (c) $[\mathrm{S} 13, \mathrm{~S} 14, \ldots, \mathrm{S} 18]$.

Figure 9 illustrates the radiation pattern obtained using CST Microwave Studio and HFSS simulations for the configuration using cophasal subarrays and the circular patch in scanning directions of $\theta_{0}=-40^{\circ},-30^{\circ}, 20^{\circ}$ and $40^{\circ}$. As shown in Figure 9, the side-lobe level response was maintained at low values, i.e., a side-lobe level of $-19.76 \mathrm{~dB}$ with CST and $-18.75 \mathrm{~dB}$ with HFSS at the most distant scanning directions considering the antenna element based in the circular patch with the characteristics mentioned previously. The two pattern responses (obtained using CST and HFSS) are very similar.
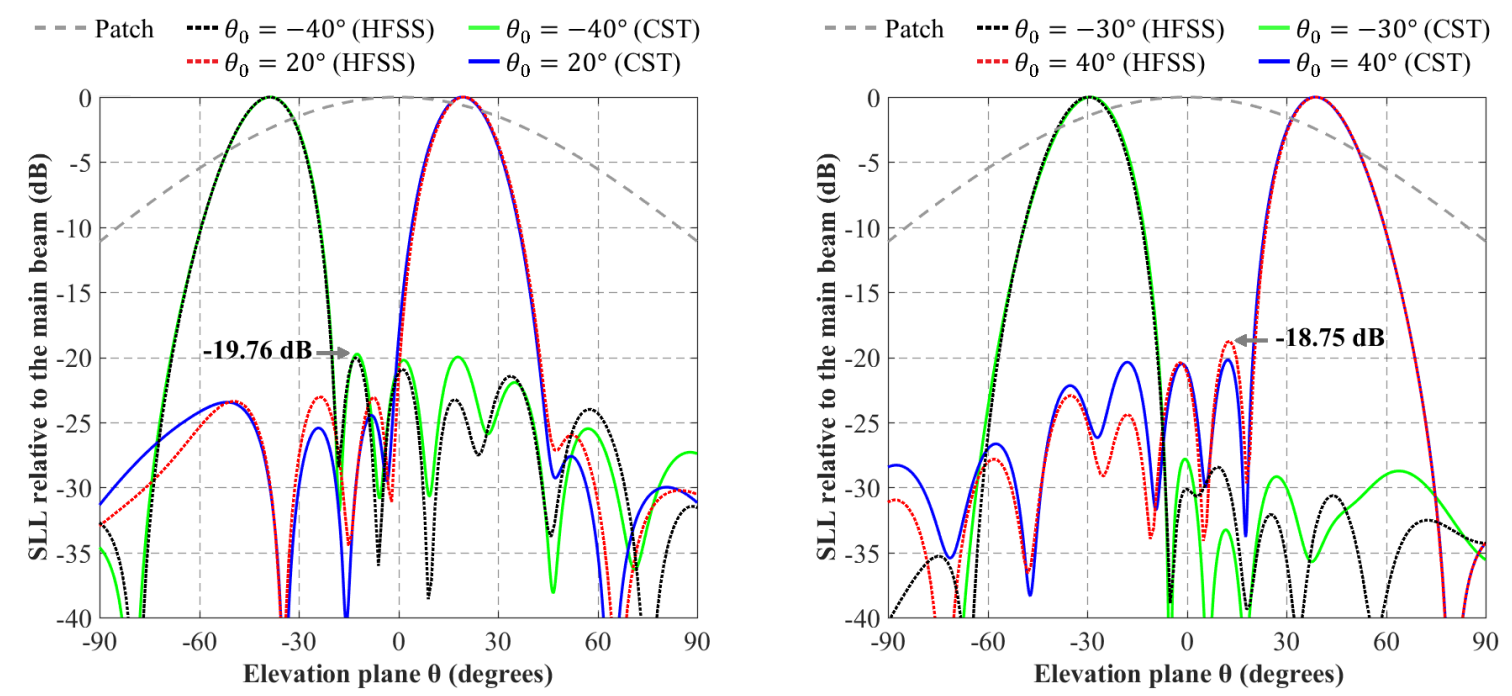

Figure 9. Radiation pattern obtained using CST and HFSS simulations for the configuration using cophasal subarrays for the case optimized by differential evolution.

Figure 10 shows the array configuration using circular patches and the 3D radiation pattern obtained using CST Microwave Studio simulation for the case using cophasal subarrays in scanning 
directions of $\theta_{0}=-40^{\circ}, \theta_{0}=0^{\circ}$ and $\theta_{0}=40^{\circ}$. These simulation results demonstrate that for the proposed configuration of concentric rings array using cophasal subarrays for grouping the elements, the technique of differential evolution shows that the values of the amplitude and phase excitations feeding each cophasal subarray provide a low side-lobe level.

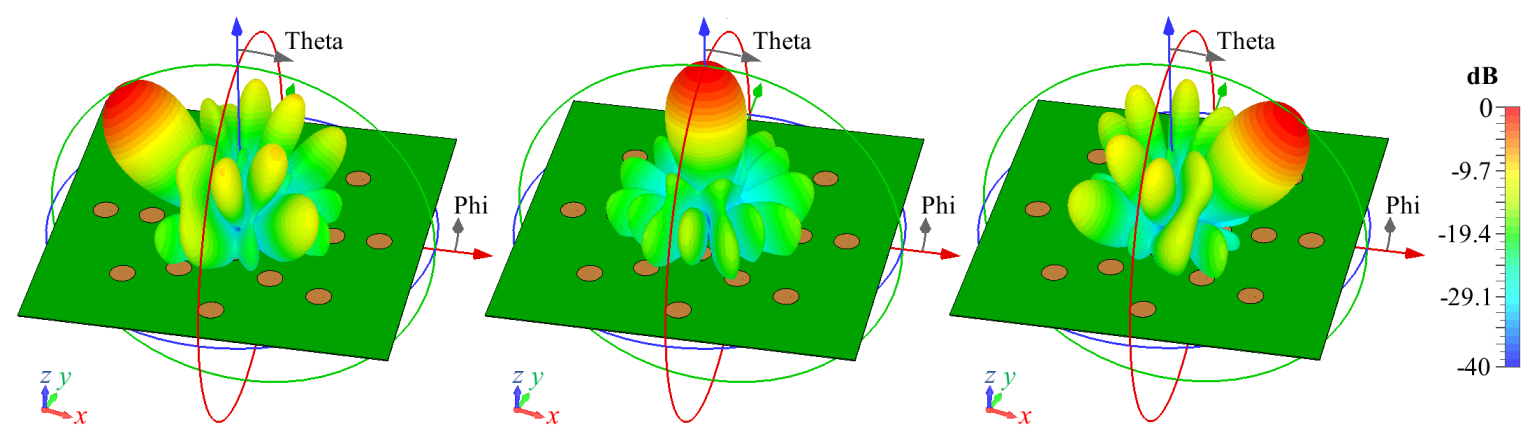

Figure 10. 3D Radiation pattern obtained using CST Microwave Studio simulation for the configuration using cophasal subarrays (and the circular patch) for the case optimized by differential evolution (scanning directions in $\theta_{0}=-40^{\circ}, \theta_{0}=0^{\circ}$ and $\theta_{0}=40^{\circ}$ ).

Furthermore, a better side lobe level performance was obtained with a significant reduction in the number of phase shifters compared to a conventional array (that uses one phase shifter per antenna element without optimization). The CST Microwave Studio simulations using the circular patch as antenna element generated a scannable array pattern over the elevation plane in the range $\left[-40^{\circ}, 40^{\circ}\right]$ without pattern distortion during beam steering.

Table 1 shows a comparison of the CST simulation results (directivity and side-lobe level) with respect to the results without considering mutual coupling. Mutual coupling does not deteriorate the pattern features obtained. The minimum spacing is $0.5 \lambda$ among antenna elements. This helps to avoid degradations caused by electromagnetic couplings.

Table 1. Comparison of the CST simulation results (directivity and side-lobe level) with respect to the theoretical results for the analyzed scanning angles.

\begin{tabular}{|c|c|c|c|c|}
\hline \multirow{2}{*}{$\begin{array}{l}\text { Main Beam Direction } \\
\qquad\left(\theta_{0}\right)\end{array}$} & \multicolumn{2}{|c|}{ Array Factor } & \multicolumn{2}{|c|}{ CST } \\
\hline & $\begin{array}{l}\text { Side Lobe Level } \\
\text { (dB) }\end{array}$ & $\begin{array}{l}\text { Directivity } \\
\text { (dB) }\end{array}$ & $\begin{array}{l}\text { Side Lobe Level } \\
\text { (dB) }\end{array}$ & $\begin{array}{l}\text { Directivity } \\
\text { (dB) }\end{array}$ \\
\hline$-40^{\circ}$ & -20.29 & 11.99 & -19.76 & 12.23 \\
\hline$-35^{\circ}$ & -25.86 & 12.04 & -24.26 & 12.14 \\
\hline$-30^{\circ}$ & -26.87 & 12.22 & -27.83 & 12.28 \\
\hline$-25^{\circ}$ & -25.34 & 12.48 & -26.79 & 12.57 \\
\hline$-20^{\circ}$ & -22.99 & 12.60 & -23.40 & 12.67 \\
\hline$-15^{\circ}$ & -22.68 & 12.63 & -23.38 & 12.68 \\
\hline$-10^{\circ}$ & -23.31 & 12.61 & -23.78 & 12.61 \\
\hline$-5^{\circ}$ & -24.18 & 12.70 & -23.01 & 12.70 \\
\hline $0^{\circ}$ & -25.17 & 12.72 & -25.77 & 12.73 \\
\hline $5^{\circ}$ & -24.18 & 12.70 & -23.01 & 12.70 \\
\hline $10^{\circ}$ & -23.31 & 12.61 & -23.83 & 12.61 \\
\hline $15^{\circ}$ & -22.68 & 12.63 & -23.42 & 12.67 \\
\hline $20^{\circ}$ & -22.99 & 12.60 & -23.46 & 12.67 \\
\hline $25^{\circ}$ & -25.34 & 12.48 & -26.82 & 12.57 \\
\hline $30^{\circ}$ & -26.87 & 12.22 & -28.18 & 12.29 \\
\hline $35^{\circ}$ & -25.86 & 12.04 & -24.52 & 12.14 \\
\hline $40^{\circ}$ & -20.29 & 11.99 & -20.21 & 12.22 \\
\hline
\end{tabular}

Furthermore, Table 2 shows a performance comparison of the design case for the geometry of concentric rings with respect to other state-of-the-art array geometries. This comparison is achieved 
in terms of the array geometry, number of antenna elements, reduction of phase shifters, scanning range and side lobe level. The case of cophasal subarrays presents a reduction in phase shifters of $66 \%$ for a scanning range of $\pm 40^{\circ}$ with a peak side-lobe level of $-20 \mathrm{~dB}$. The case of cophasal subarrays outperforms all cases in terms of the scanning capacity and side-lobe level, and resulting in a good compromise in the reduction of phase shifters by $66 \%$.

Table 2. A performance comparison of the design case based in cophasal subarrays for the geometry of concentric rings with respect to other array geometries published in state of art.

\begin{tabular}{|c|c|c|c|c|c|c|c|}
\hline & \multicolumn{2}{|c|}{ Geometry } & $\begin{array}{l}\text { Number } \\
\text { of } \\
\text { Elements }\end{array}$ & $\begin{array}{l}\text { Number } \\
\text { of Phase } \\
\text { Shifters }\end{array}$ & $\begin{array}{c}\text { Reduction of } \\
\text { Phase } \\
\text { Shifters (\%) }\end{array}$ & $\begin{array}{l}\text { Scanning } \\
\text { Range }\end{array}$ & $\begin{array}{c}\text { Peak Sidel } \\
\text { Lobe } \\
\text { Level }\end{array}$ \\
\hline $\begin{array}{l}\text { Cophasal } \\
\text { subarrays }\end{array}$ & \multicolumn{2}{|c|}{ Concentric rings } & 18 & 6 & $66 \%$ & $\pm 40^{\circ}$ & $-20 \mathrm{~dB}$ \\
\hline Conventional case & \multicolumn{2}{|c|}{ Concentric rings } & 18 & 18 & $0 \%$ & $\pm 39^{\circ}$ & $-15 \mathrm{~dB}$ \\
\hline $\begin{array}{c}\text { Uniformly } \\
\text { subarrays [2] }\end{array}$ & \multicolumn{2}{|c|}{ Linear array } & 36 & 12 & $66 \%$ & $\pm 6^{\circ}$ & $-15 \mathrm{~dB}$ \\
\hline \multirow{3}{*}{$\begin{array}{l}\text { Overlapped } \\
\text { subarrays }\end{array}$} & \multirow{3}{*}{$\begin{array}{l}\text { Planar } \\
\text { array }\end{array}$} & [3] & 80 & 4 & $95 \%$ & $\pm 7^{\circ}$ & $-20 \mathrm{~dB}$ \\
\hline & & \multirow{2}{*}{ [20] } & 28 & 14 & $50 \%$ & $\pm 24^{\circ}$ & $-15 \mathrm{~dB}$ \\
\hline & & & 28 & 7 & $75 \%$ & $\pm 11^{\circ}$ & $-15 \mathrm{~dB}$ \\
\hline $\begin{array}{l}\text { Randomly feeding } \\
\text { network [2] }\end{array}$ & \multicolumn{2}{|c|}{ Linear array } & 30 & 12 & $60 \%$ & $\pm 14^{\circ}$ & $-15 \mathrm{~dB}$ \\
\hline \multirow{2}{*}{ CORPS [33] } & \multirow{2}{*}{\multicolumn{2}{|c|}{ Linear array }} & 10 & 8 & $20 \%$ & $\pm 30^{\circ}$ & $-19 \mathrm{~dB}$ \\
\hline & & & 10 & 7 & $30 \%$ & $\pm 20^{\circ}$ & $-16 \mathrm{~dB}$ \\
\hline
\end{tabular}

\subsection{Manufacturing Tolerances}

An analysis of the manufacturing tolerances was made to verify the reliability of our design, i.e., the control points (the design variables: amplitude and phase excitations) can differ from the ideal because of manufacturing tolerances [34]. Tolerances in the implementation of the amplitude levels and the phase shifts (produced by the phase shifters) could result in a pattern with relatively high side-lobe level [34]. Therefore, errors in the amplitude and phase values of the excitations were taken into account by means of a probability distribution function where random and statistically independent errors among the array elements were assumed [35], i.e., we can model the manufacturing tolerance errors by considering the amplitude and phase errors as statistically independent functions. These error functions can be modelled by a Rician probability function [35].

Figure 11 shows the error intervals for the ideal values of the amplitude and phase excitations (obtained for main lobe direction of $\theta_{0}=-40^{\circ}$ ) considering a tolerance of $5 \%$. Figure 11 illustrates that the error is directly proportional to the value of the amplitude and phase excitation, i.e., we have a higher value of error for higher values of amplitude and phase. The maximum error interval is found in the antenna element $n_{15}$ for the amplitude excitation (from 10.43 to $11.52, \pm 0.54$ ), and, in the elements $n_{11}$ and $n_{15}$ for the phase $\left( \pm 0.32\right.$ radians $\left.\approx \pm 18^{\circ}\right)$. 


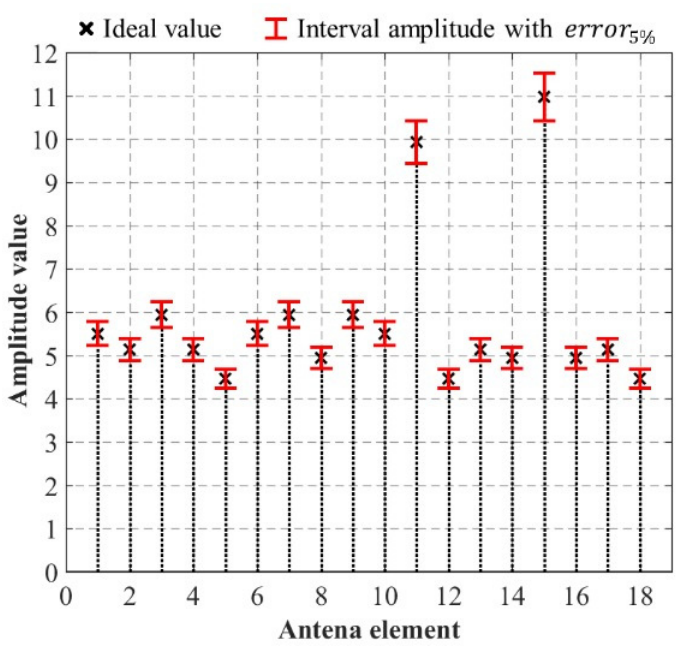

(a)

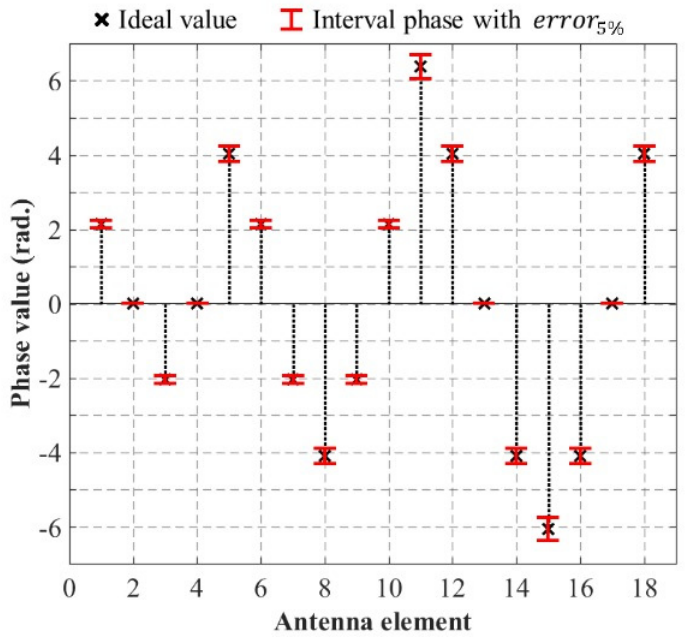

(b)

Figure 11. Error interval for the values of the amplitude (a) and phase (b) excitations with a tolerance of $5 \%$ and considering a scanning direction in $\theta_{0}=-40^{\circ}$.

All scanning directions were examined using 300 iterations with a tolerance of $1 \%$ and $5 \%$. Here we illustrate the cases of scanning directions more sensitive to the tolerance errors. Figure 12 illustrates the response of the array factor for all the iterations obtained using the amplitude and phase values with an error tolerance of $1 \%$ (Figure 12a) and $5 \%$ (Figure 12b) for a scanning direction in $\theta_{0}=-40^{\circ}$, and for a scanning direction in $\theta_{0}=20^{\circ}$ (Figure 12c) with $5 \%$ tolerance. As shown in Figure 12, the side-lobe level response remained below a peak value of $-17.04 \mathrm{~dB}$ for the most sensitive case.

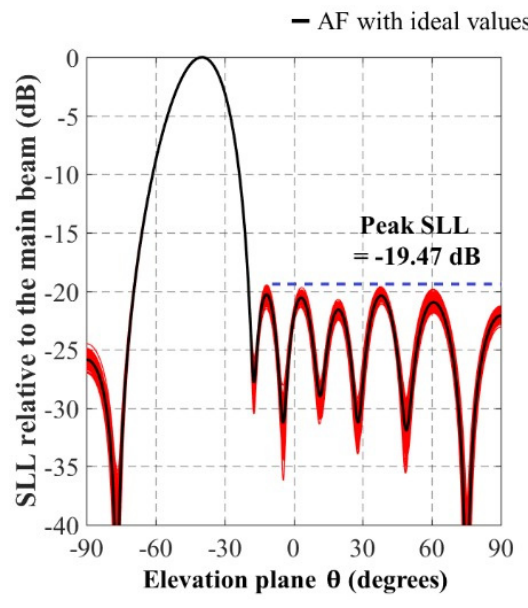

(a)

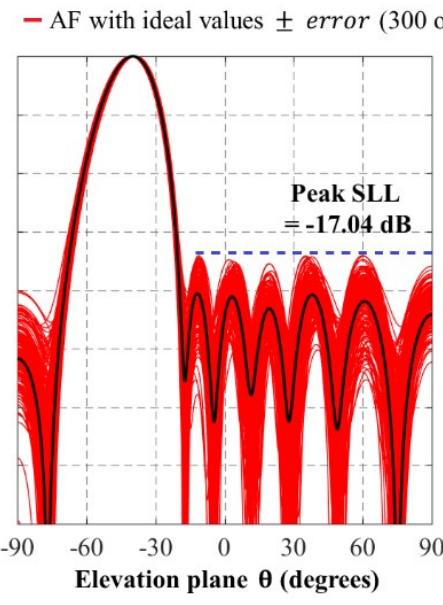

(b)

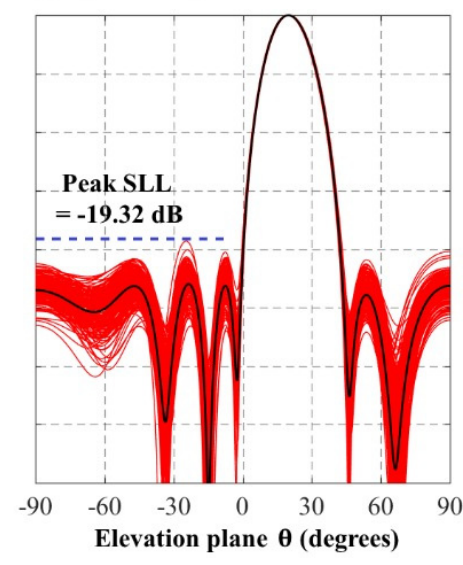

(c)

Figure 12. Array factor response for 300 iterations obtained by using the amplitude and phase values with an error tolerance of $1 \%(\mathbf{a})$ and $5 \%$ (b) for a scanning direction in $\theta_{0}=-40^{\circ}$, and for a scanning direction in $\theta_{0}=20^{\circ}$ (c) with $5 \%$ of tolerance.

Figure 13 shows an analysis of the manufacturing tolerances using ElectroMagnetic software. The response of the array pattern (considering the iterations corresponding to the lowest and the highest values of side-lobe level) is illustrated using CST Microwave Studio, and their comparison with respect to the results without considering mutual coupling. As expected, the most sensitive case achieved a peak value of $-17.04 \mathrm{~dB}$ and a misalignment of $2^{\circ}$ for the main beam. Therefore, the design values obtained can be considered reliable considering manufacturing tolerances. 

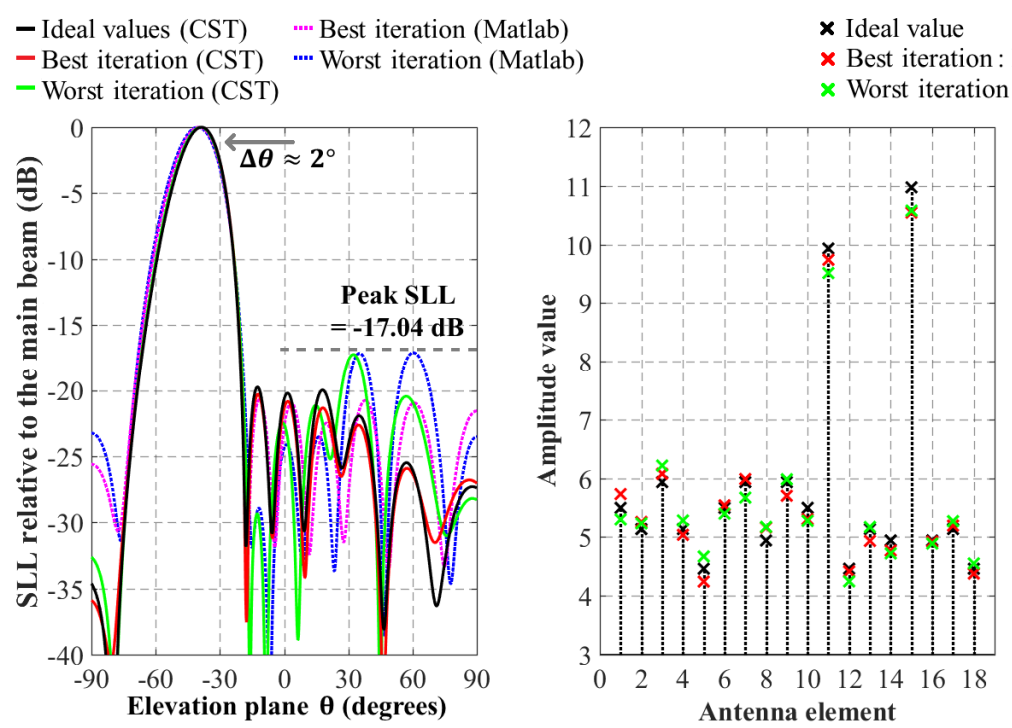

ideal value \pm error $_{5 \%}$
ion: Ideal value \pm error $_{5 \%}$

Figure 13. Analysis of the manufacturing tolerances using CST Microwave Studio.

Although only the configuration of concentric rings array was analyzed in this paper, other array configurations could be analyzed using cophasal subarrays for reducing the number of phase shifters used in antenna systems.

\section{Conclusions}

The results derived using CST Microwave Studio illustrated that the design methodology for a concentric rings array based on cophasal subarrays for grouping the elements reduces the number of phase shifters by $66 \%$ for a scanning range of $\pm 40^{\circ}$ with a peak side-lobe level of $-20 \mathrm{~dB}$. The case of cophasal subarrays outperforms previous work cases in scanning capacity and side-lobe level, and results in a good compromise in terms of the reduction of the number of phase shifters. The proposed approach obtained a better side-lobe level performance with a reduction in the number of phase shifters compared to a conventional array (that uses one phase shifter per antenna element without optimization). Furthermore, an analysis of the manufacturing tolerances was made in EM software to verify the reliability of our design. The most sensitive case achieved a peak value of $-17.04 \mathrm{~dB}$, therefore, the design values obtained can be considered reliable considering manufacturing tolerances.

Future works will deal with other antenna array configurations using cophasal subarrays for reducing the number of phase shifters used in antenna systems.

Author Contributions: Conceptualization, M.A.P.; methodology, E.J.; software, A.R.; validation, M.A.P. and D.H.C.; formal analysis, A.M.; investigation, M.A.P. and E.J.; resources, E.J. and M.A.P.; data curation, E.M.; writing-original draft preparation, M.A.P., E.J. and D.H.C.; writing-review and editing, E.M.; visualization, A.M.; supervision, M.A.P.; project administration, M.A.P. and D.H.C.; funding acquisition, M.A.P. All authors have read and agreed to the published version of the manuscript.

Funding: This paper has been supported by the Mexican Council for Science and Technology (CONACyT), under Grant no. 2016-01-1680.

Conflicts of Interest: The authors declare no conflict of interest.

\section{References}

1. Rebeiz, G.M.; Tan, G.; Hayden, J.S. RF MEMS phase shifters. IEEE Microw. Mag. 2002, 3, 72-81. [CrossRef]

2. Avser, B.; Pierro, J.; Rebeiz, G.M. Random feeding networks for reducing the number of phase shifters in limited-scan arrays. IEEE Trans. Antennas Propag. 2016, 64, 4648-4658. [CrossRef]

3. Abbaspour-Tamijani, A.; Sarabandi, K. An affordable millimeter-wave beam-steerable antenna using interleaved planar subarrays. IEEE Trans. Antennas Propag. 2003, 51, 2193-2202. [CrossRef] 
4. Toyama, N. Aperiodic array consisting of subarrays for use in small mobile earth station. IEEE Trans. Antennas Propag. 2005, 53, 2004-2010. [CrossRef]

5. Wang, D.; Hu, H.; Yang, Z. Improved genetic algorithm for the configuration optimization of the sub arrays in phased array radar. In Proceedings of the 9th International Congress on Image and Signal. Processing, BioMedical Engineering and Informatics (CISP-BMEI), Datong, China, 15-17 October 2016; pp. 930-934.

6. Wincza, K.; Gruszczynski, S. Reduced sidelobe low-cost antenna array with corner-coupled patches for radar applications. In Proceedings of the Asia-Pacific Microwave Conference, Melbourne, Australia, 5-8 December 2011; pp. 1202-1205.

7. Wang, D.; Hu, H.; Yang, Z. The pareto rank algorithm for the division of the subarrays for the phase array radar. In Proceedings of the 9th International Congress on Image and Signal Processing, BioMedical Engineering and Informatics (CISP-BMEI), Datong, China, 15-17 October 2016; pp. 945-949.

8. Schneider, M. Automotive radar-status and trends. In Proceedings of the German Microwave Conference, Hildesheim, Germany, 5-7 April 2005; pp. 144-147.

9. Bui, L.Q.; Franklin, M.R.; Taylor, C.; Neilson, G. Autonomous landing guidance system validation. In Proceedings of the International Society for Optics and Photonics, Orlando, FL, USA, 26 June 1997; pp. 19-25.

10. Haupt, R.L. Optimized Weighting of Uniform Subarrays of Unequal Sizes. IEEE Trans. Antennas Propag. 2007, 55, 1207-1210. [CrossRef]

11. Rocca, P.; Haupt, R.L.; Massa, A. Sidelobe Reduction through Element Phase Control in Uniform Subarrayed Array Antennas. IEEE Antennas Wirel. Propag. Lett. 2009, 8, 437-440. [CrossRef]

12. Haupt, R. Optimization of subarray amplitude tapers. In Proceedings of the IEEE Antennas and Propagation Society International Symposium, 1995 Digest, Newport Beach, CA, USA, 18-23 June 1995; pp. 1830-1833.

13. Mailloux, R.J.; Santarelli, S.G.; Roberts, T.M.; Luu, D. Irregular polyomino-shaped subarrays for space-based active arrays. Int. J. Antennas Propag. 2009, 2009, 1-9. [CrossRef]

14. Wang, H.; Fang, D.G.; Chow, Y.L. Grating lobe reduction in a phased array of limited scanning. IEEE Trans. Antennas Propag. 2008, 56, 1581-1586. [CrossRef]

15. Azar, T. Overlapped subarrays: Review and update. IEEE Antennas Propag. Mag. 2013, 55, $228-234$. [CrossRef]

16. Mailloux, R.J. A low-sidelobe partially overlapped constrained feed network for time-delayed subarrays. IEEE Trans. Antennas Propag. 2001, 49, 280-291. [CrossRef]

17. Herd, J.S.; Duffy, S.M.; Steyskal, H. Design considerations and results for an overlapped subarray radar antenna. In Proceedings of the 2005 IEEE Aerospace Conference, Big Sky, MT, USA, 5-12 March 2005; pp. 1087-1092.

18. Duffy, S.M.; Santiago, D.D.; Herd, J.S. Design of overlapped subarrays using an RFIC beamformer. In Proceedings of the 2007 IEEE Antennas and Propagation Society International Symposium, Honolulu, HI, USA, 9-15 June 2007; pp. 1949-1952.

19. Mailloux, R.J. An overlapped subarray for limited scan application. IEEE Trans. Antennas Propag. 1974, 22, 487-489. [CrossRef]

20. Avser, B.; Frazita, R.F.; Rebeiz, G.M. Interwoven feeding networks with aperture sinc-distribution for limited-scan phased arrays and reduced number of phase shifters. IEEE Trans. Antennas Propag. 2018, 66, 2401-2413. [CrossRef]

21. Krivosheev, Y.V.; Shishlov, A.V.; Denisenko, V.V. Grating lobe suppression in aperiodic phased array antennas composed of periodic subarrays with large element spacing. IEEE Antennas Propag. Mag. 2015, 57, 76-85. [CrossRef]

22. Price, K.V.; Storn, R.M.; Lampinen, J.A. Differential Evolution: A Practical Approach to Global Optimization; Springer: Berlin, Germany, 2005.

23. Stearns, C.O.; Stewart, A.C. An investigation of concentric ring antennas with low sidelobes. IEEE Trans. Antennas Propag. 1965, 18, 856-863. [CrossRef]

24. Haupt, R.L. Optimized element spacing for low sidelobe concentric ring arrays. IEEE Trans. Antennas Propag. 2008, 56, 266-268. [CrossRef]

25. Ibarra, M.; Panduro, M.A.; Andrade, A. Differential Evolution Multi-Objective for Optimization of Isoflux Antenna Arrays. Iete Tech. Rev. 2016, 33, 105-114. [CrossRef] 
26. Elizarraras, O.; Panduro, M.A.; Mendez, A.; Reyna, A.; Covarrubias, D. Design of Circular Antenna Arrays of Circular Subarrays Exploiting Rotational Symmetry. J. Electromagn. Waves Appl. 2017, 31, 1277-1288. [CrossRef]

27. Ibarra, M.; Panduro, M.A.; Andrade, A.; Reyna, A. Design of Sparse Concentric Rings Array for LEO Satellites. J. Electromagn. Waves Appl. 2015, 29, 1983-2001. [CrossRef]

28. Garza, L.A.; Panduro, M.A.; Covarrubias, D.H.; Reyna, A. Multi-objective Synthesis of Steerable UWB Circular Antenna Array considering Energy Patterns. Int. J. Antennas Propag. 2015, 2015, 789094. [CrossRef]

29. Medina, Z.; Reyna, A.; Panduro, M.A.; Elizarraras, O. Dual-Band Performance Evaluation of Time-Modulated Circular Geometry Array with Microstrip-Fed Slot Antennas. IEEE Access 2019, 7, 28625-28634. [CrossRef]

30. Panduro, M.A.; Reyna, A.; Covarrubias, D.H. Non-Uniform Concentric Rings Design for Ultra-Wideband Arrays. Sensors 2019, 19, 2262. [CrossRef]

31. Balanis, C. Antenna Theory-Analysis and Design, 2nd ed.; Willey: New York, NY, USA, 1997.

32. Kahn, W. Active reflection coefficient and element efficiency in arbitrary antenna arrays. IEEE Trans. Antennas Propag. 1969, 17, 653-654. [CrossRef]

33. Panduro, M.A.; del Rio-Bocio, C. Design of beamforming networks using CORPS and evolutionary optimization. AEU Int. J. Electron. Commun. 2009, 63, 353-365. [CrossRef]

34. Hsiao, J.K. Design of error tolerance of a phased array. Electron. Lett. 1985, 21, 834-836. [CrossRef]

35. Anselmi, N.; Manica, L.; Rocca, P.; Massa, A. Tolerance Analysis of Antenna Arrays through Interval Arithmetic. IEEE Trans. Antennas Propag. 2013, 61, 5496-5507. [CrossRef]

(C) 2020 by the authors. Licensee MDPI, Basel, Switzerland. This article is an open access article distributed under the terms and conditions of the Creative Commons Attribution (CC BY) license (http://creativecommons.org/licenses/by/4.0/). 